\title{
BMJ Open Efficacy of positive psychotherapy in reducing negative and enhancing positive psychological outcomes: a meta-analysis of randomised controlled trials
}

\author{
Thole Hilko Hoppen (D), Nexhmedin Morina (D)
}

To cite: Hoppen TH, Morina N. Efficacy of positive psychotherapy in reducing negative and enhancing positive psychological outcomes: a meta-analysis of randomised controlled trials. BMJ Open 2021;11:e046017. doi:10.1136/ bmjopen-2020-046017

- Prepublication history and additional supplemental material for this paper are available online. To view these files, please visit the journal online. (http://dx.doi.org/10.1136/ bmjopen-2020-046017).

Received 19 0ctober 2020 Accepted 09 August 2021

D Check for updates

(c) Author(s) (or their employer(s)) 2021. Re-use permitted under CC BY-NC. No commercial re-use. See rights and permissions. Published by BMJ.

Institute of Psychology, University of Münster, Münster, Germany

Correspondence to Dr Thole Hilko Hoppen; thoppen@uni-muenster.de

\section{ABSTRACT}

Objective Positive psychotherapy (PPT) aims at increasing positive affect, meaning and engagement. We aimed to synthesise the available evidence on PPT efficacy. Design We conducted a preregistered systematic literature search and meta-analysis of randomised controlled trials examining the efficacy of PPT for increasing positive (eg, satisfaction with life) or decreasing negative psychological outcomes (eg, depression). Data sources Medline, PsycINFO and Web of Science from 2006 (ie, inception of PPT) to February 2020 as well as related systematic reviews and meta-analyses. Results We included 20 randomised controlled trial with a total of 1360 participants. Moderate effect sizes were found for increasing positive outcomes $(\mathrm{g}=-0.72$, $95 \% \mathrm{Cl}:-1.31$ to $-0.14, \mathrm{k}=10$, numbers needed to treat $(\mathrm{NNT})=2.55)$ and reducing negative outcomes $(\mathrm{g}=0.48$, 95\% Cl: 0.18 to $0.78, \mathrm{k}=8$, NNT=3.76) when PPT was compared with waitlist control conditions at posttreatment assessment. When compared with active control conditions, PPT yielded large effect sizes for increasing positive outcomes $(\mathrm{g}=-0.92,95 \% \mathrm{Cl}:-1.74$ to -0.11 , $\mathrm{k}=6, \mathrm{NNT}=2.05)$ and reducing depression $(\mathrm{g}=0.94,95 \%$ Cl: 0.18 to $1.70, k=6, N N T=2.03$ ) at post-treatment assessment. No significant differences in efficacy were found when compared with established treatments such as cognitive-behavioural therapy. Evidence was found to support an association between trial quality and effect sizes. For positive outcomes, higher trial quality was related to larger effect size. Whereas higher trial quality was associated with smaller effect size for depression. Follow-up assessments remained too scarce for most planned analyses.

Conclusions Our findings support the short-term efficacy of PPT. However, results are to be regarded with due caution in the light of low number of trials. More highquality trials that assess efficacy at follow-ups are needed to draw firmer conclusions on the long-term efficacy of PPT.

PROSPERO registration number CRD42020173567.

\section{INTRODUCTION}

Positive psychotherapy (PPT) is theoretically grounded in the field of positive psychology

\section{STRENGTHS AND LIMITATIONS OF THIS STUDY}

$\Rightarrow$ This meta-analysis was preregistered and conducted in line with the Preferred Reporting Items for Systematic Reviews and Meta-analyses guidelines.

$\Rightarrow$ Data synthesis was based on a broad systematic literature search including broad secondary manual searches.

$\Rightarrow$ Potential moderators including trial quality, treatment lengths and alliance were analysed.

$\Rightarrow$ Scarcity of available trials precluded many (sub) analyses and asks for due caution in interpreting the present findings.

$\Rightarrow$ Due to lacking follow-up assessment, long-term efficacy could not be determined.

and proposes that psychopathology such as depression can be effectively treated by directly and primarily building and strengthening pleasure (ie, positive emotions), meaning (ie, belonging to and serving something greater than the self) and engagement (ie, active involvement in daily life). ${ }^{1}$ PPT presumes that by means of fostering positive resources, negative symptoms will be successfully dampened. While the founders believed from inception that PPT might be an effective treatment for various disorders, they started off by investigating its efficacy in treating depression. PPT consists of single positive interventions such as Using Your Strength, the Three Good Things and the Gratitude Visit. In Using Your Strength, for instance, participants are asked to fill out the Values in Action Inventory of Strengths ${ }^{2}$ and to think of ways to use their top five strengths more in daily life. Seligman et al ended up including 26 positive exercises in their final PPT manual. In their first randomised controlled trial (RCT) on the efficacy of PPT, they offered a 6-week, 2-hour per week group intervention with 8-11 mildly to moderately depressed students 
per group and found that PPT was effective in lowering depressive symptoms and increasing satisfaction with life compared with waitlist controls. ${ }^{1}$ They also conducted a second RCT were they offered a 14-session individual PPT over 12 weeks in a sample of adults suffering from major depressive disorder. Again, PPT was found effective in decreasing depression and increasing happiness, in this RCT compared with treatment-as-usual. ${ }^{1}$ Since then, numerous other RCTs have assessed the efficacy of PPT. ${ }^{3}$ Apart from further research on populations suffering depressive symptoms or depressive disorders, PPT has been investigated in various other contexts including patients with psychosis ${ }^{4}$ and multiple other mental disorders $^{5}$ as well as in patients with several somatic complaints such as cancer ${ }^{67}$ or multiple sclerosis. ${ }^{8}$ In their systematic review of the PPT literature, Walsh et al summarised the findings of 12 publications (from 9 individuals trials) published before May 2015. ${ }^{3}$ The authors conclude that the application of PPT in intervention research is heterogeneous in terms of both, the modifications of the original manual as well as the conditions targeted by PPT as intended by the PPT developers. ${ }^{19}$ To the best of our knowledge, no meta-analysis with an exclusive focus on the efficacy of PPT has been published to this date. Against this background, we performed a systematic literature review and meta-analysis of RCTs assessing the efficacy of PPT.

\section{METHODS}

Following the recommendations by the Preferred Reporting Items for Systematic Reviews and Meta-analyses group,${ }^{10}$ we defined the main structured research question describing the Population, Intervention, Comparison, Outcome and Study design as 'In individuals with mental or physical health complaints, does PPT (I), compared with control conditions (C), improve psychological outcomes $(\mathrm{O})$ in RCTs $(\mathrm{S})$ ?'.

\section{Patient and public involvement}

Not applicable. We performed a meta-analysis on published data.

\section{Literature search strategy}

Inclusion criteria for the meta-analysis consisted of: (1) RCT, (2) evaluation of the efficacy of PPT as developed by Seligman $e t a l^{l}$ and (3) a minimum of 10 participants per treatment arm at post-treatment assessment with available data on at least one relevant outcome. No restrictions were placed on age of participants, comparison conditionor publication type. Studies that only applied a mixture of PPT with another intervention, such as a mixture of PPT and cognitive-behavioural therapy (CBT) in comparison to a control condition, ${ }^{9}$ were excluded due to our narrow focus on the efficacy of PPT, as founded by Seligman et al. ${ }^{1}$ We searched the following databases: PsycINFO, MEDLINE and Web of Science from 2006 up to 13 February 2020. The year 2006 represents the year where the theoretical underpinnings of the PPT were first published. ${ }^{1}$ No other limits or filters were applied. MeSH terms for Ebscohost (regarding MEDLINE and PsycINFO) were as follows: 'SU positive psychotherapy OR TI positive psychotherapy $\mathrm{OR} A B$ positive psychotherapy' (see also online supplemental materials eList 1). In Web of Science, a similar search string to Ebscohost was chosen to search for 'positive psychotherapy' in titles, abstracts and keywords. To retrieve additional publications, the reference lists of all included papers and relevant (ie, related) meta-analyses and systematic reviews were manually screened. ${ }^{11-19}$ Secondary handsearches were conducted using Google Scholar. The study synthesis was performed independently by both authors.

\section{Coding of studies}

The publications were independently coded by both authors. From each publication, the following study, intervention and participant characteristics were coded and extracted: country the trial was conducted in, clinical population targeted (ie, any physical or mental health condition), experimental intervention type (ie, original PPT manual or modified version), intervention format (ie, individual or group), comparison group(s), session number and session duration in minutes, follow-up duration in months for the longest reported follow-up assessment of the relevant outcome(s), number of participants at post-treatment assessment, age of participants (ie, mean and SD or range), proportion of sample with female sex in per cent, applied statistical analysis (ie, completer or intention-to-treat (ITT) analyses) and relevant outcome(s) targeted by PPT. The post-treatment assessment experimental group and control group means, SD and sample sizes on the relevant outcome(s) (see in more detail below) were extracted. When reported, follow-up assessment data on relevant outcomes per group were also extracted. When multiple follow-up assessments were reported, the data from the longest follow-up assessment were retrieved. When relevant data were not reported, it was either calculated from given data (eg, SD from SEs) or the corresponding author of the respective publication was contacted via email twice with 1 month in between. In one case, we contacted authors due to unusual results. Mohamadi et al potentially reported the means and SDs for a relevant outcome (ie, quality of life) in wrong order (ie, means where SDs should be placed and vice versa). ${ }^{20}$ We contacted the authors twice via email and were left with no response. Consequently, we calculated two analyses; one with changed order of means and SD and one with unchanged order.

We divided control conditions into passive control conditions, which turned out to exclusively consist of waitlist control conditions (WLC), active control conditions (ie, treatment-as-usual and placebo exercises) and other active treatment conditions (ie, CBT, dialectic behavioural therapy (DBT) and Mindfulness-Based CBT $(\mathrm{MBCT}))$. Note that included trials included different 
physical or mental health conditions and, therefore, TAU may involve various different treatment regimens.

\section{Quality assessment}

Both authors independently rated the quality of the included trials by using a quality assessment constructed by Cuijpers $e t$ al and adjusted in two subsequent metaanalyses. ${ }^{21-23}$ After independent rating, regular digital meetings were held to discuss disagreements. This scale assesses the following nine quality criteria: (1) Were symptoms/diagnoses assessed with a semistructured diagnostic interview?, (2) Was a treatment manual used?, (3) Were therapists trained either specifically for the study or in a general training?, (4) Was treatment integrity checked by supervision and/or recordings and/or standardised instruments?, (5) Was data analysed with ITT analysis?, (6) Was group allocation performed with a true randomisation technique?, (7) Was randomisation done by an independent third person (or computer or sealed envelopes)?, (8) Were blinded assessors used for interviews? and (9) Were drop-outs adequately reported? Items for each of the nine quality criteria were scored on a fourpoint scale, where 3 indicates high quality (eg, a published treatment manual was used), 2 indicates limited quality (eg, an unpublished treatment manual was used), 1 indicates lack of required quality (eg, no treatment manual was used) and 0 indicates unknown (ie, required information not reported). When self-report measures were used to assess outcomes in a given trial, a score of 3 was given on the quality item concerning blinded assessments. In case of technology-based interventions, a trial received a score of 3 on the quality items concerning trained therapists and formal fidelity checks due to the technology-based standardised procedure. The nine ratings were then summed up to yield the respective trial quality sum score and used as a potential moderator in meta-regressions.

\section{Data extraction of outcome measures}

For each study, a maximum of two outcomes were selected, one positive psychological outcome (if available) and one negative (if available). Choice of extracted positive and/or negative psychological outcome(s) was data driven. That is, we first extracted all negative and positive psychological outcomes per trial and then analysed across all included trials which positive and negative psychological outcomes were most frequently assessed and reported in the PPT trial literature. For the negative outcomes, depression was by far the most frequently assessed outcome $(\mathrm{k}=14)$ and the sole negative outcome extracted. Assessment of positive outcomes was more heterogeeous. Satisfaction with life was assessed most often $(k=11)$, consecutively followed by happiness $(k=9)$, well-being $(\mathrm{k}=5)$, hope $(\mathrm{k}=5)$, positive affect $(\mathrm{k}=4)$, quality of life $(\mathrm{k}=3)$, self-efficacy $(\mathrm{k}=2)$ and meaning in life $(\mathrm{k}=1)$. As such, we prioritised satisfaction with life first in the data extraction phase when several positive outcomes were reported in a given trial, happiness second and so forth. We planned to conduct two overarching analyses across included negative and positive outcomes, respectively, as well as subanalyses on all individual outcomes with a sufficient number of independent trials (ie, $\mathrm{k} \geq 4$ ). Data were extracted by both authors and regular digital meetings were held to discuss disagreements.

\section{Statistical analysis}

Analyses were completed with the metafor package (V.1.9.8) in R V.3.5. using random-effects models given that we expected large heterogeneity in reported effect sizes ${ }^{24-26}$ We prioritised ITT data when available $(\mathrm{k}=3)$ over completer data $(\mathrm{k}=17$, including $\mathrm{k}=3$ with insufficient information on participant flow, see table 1 for further information). To obtain the effect size Hedges's $\mathrm{g}$, R first calculates the standardised mean difference d (ie, control group mean subtracted from the experimental group mean and then divided by the pooled SD). The standardised mean difference is then multiplied by a sample size correction factor $\mathrm{J}=1-(3 /(4 \mathrm{df}-1))$ to yield Hedges's g. ${ }^{27}$ Analyses were conducted if four or more trials were available for a given (sub)analysis. ${ }^{28}$ Effect sizes g may be conservatively interpreted with Cohen's convention of small $( \pm 0.2)$, medium $( \pm 0.5)$ and large $( \pm 0.8)$ effects. ${ }^{29}$ As a test of homogeneity of effect sizes, we calculated the Q-statistic and the corresponding $p$ value. We also calculated the $\mathrm{I}^{2}$ statistic, as a measure of heterogeneity of effect sizes across trials in per cent. It has been suggested that $\mathrm{I}^{2}$-statistics of 25,50 , and $75 \%$ may be interpreted as referring to low, moderate and high levels of heterogeneity, respectively. ${ }^{30}$ Because we expected large heterogeneity, we also calculated prediction intervals. ${ }^{31}$ Prediction intervals, unlike $\mathrm{I}^{2}$-statistics, present a heterogeneity estimate in the same metric as the original effect size measure (ie, g). As such, prediction intervals provide a predicted range for the true treatment effect in similar future trials. ${ }^{32}$ When the prediction interval excludes the null, it is likely that similar future trials will also find significant effects. To check for potential effects of outliers on meta-analytic outcomes, we aimed at repeating analyses without identified outliers. Outliers were defined as effect sizes departing 3.3 SD away from the pooled mean effect in both directions. ${ }^{33}{ }^{34}$ However, no outliers were identified in any of the performed analyses. When analyses consisted of at least 10 trials, ${ }^{35}$ we assessed risk of publication bias through visual inspection of funnel plots, Egger's test of asymmetry and number of missing studies using the trim-and fill procedure. ${ }^{36}$ The trim-and-fill procedure yields an asymmetry-corrected estimate of the effect size (ie, taking publication bias into account). We calculated the numbers needed to treat $(\mathrm{NNT})$ as a measure of efficacy that is easily interpretable from a clinical perspective. It informs about the numbers of patients that need to be treated until one adverse event is prevented. ${ }^{37}$ NNTs were calculated with the NNT function of the dmetar package and are based on the pooled effect sizes (ie, Hedges' g). Lastly, we performed moderator analyses in $\mathrm{R}$ with trial quality sum score and treatment length (in minutes) as continuous variables (ie, 
$\mathscr{O}$ \&

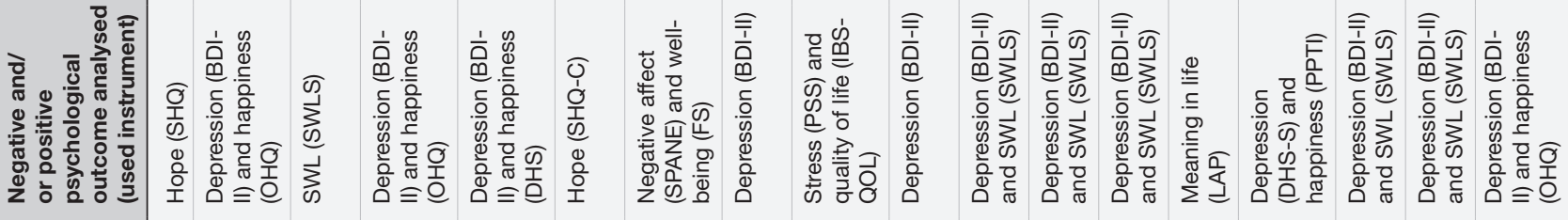

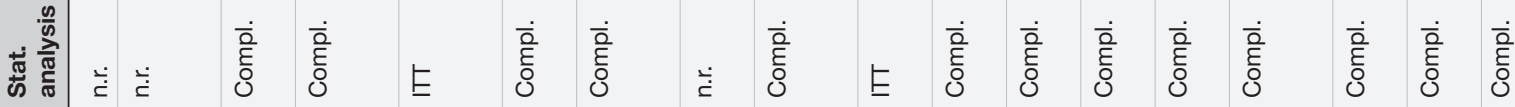

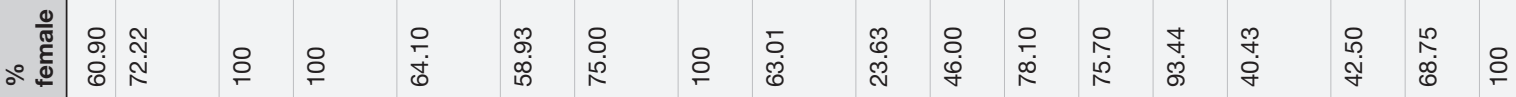

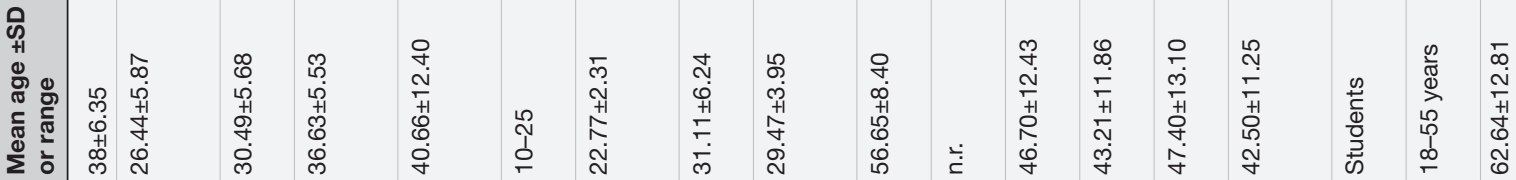

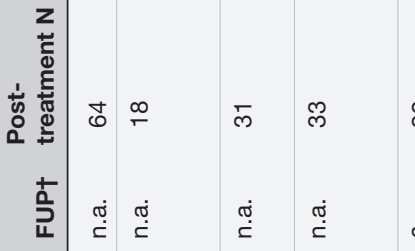

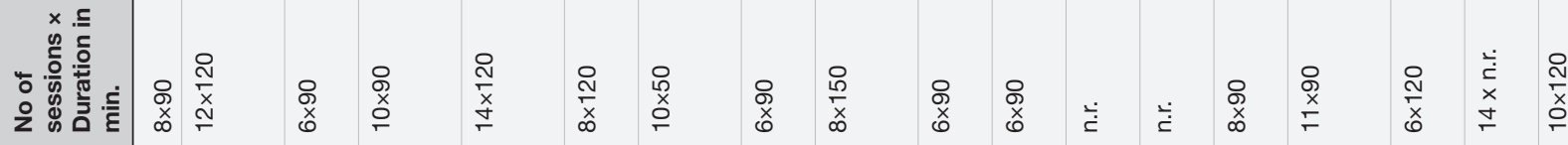

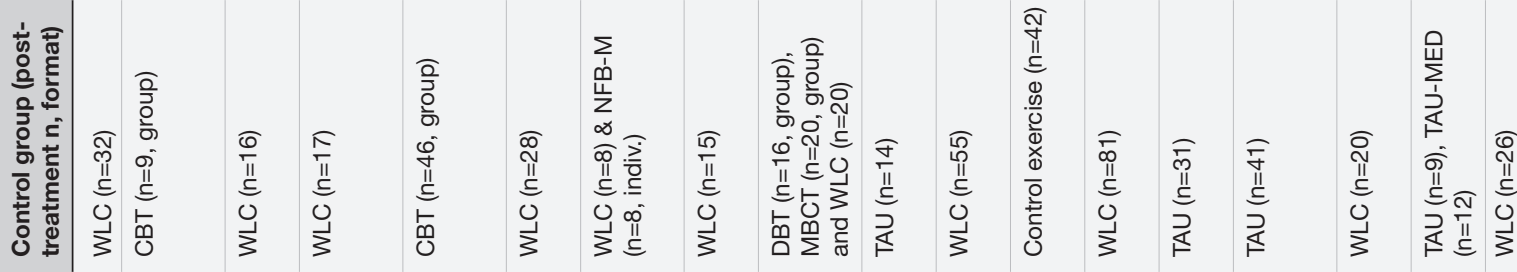

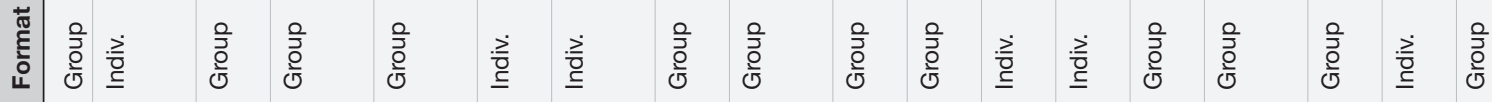

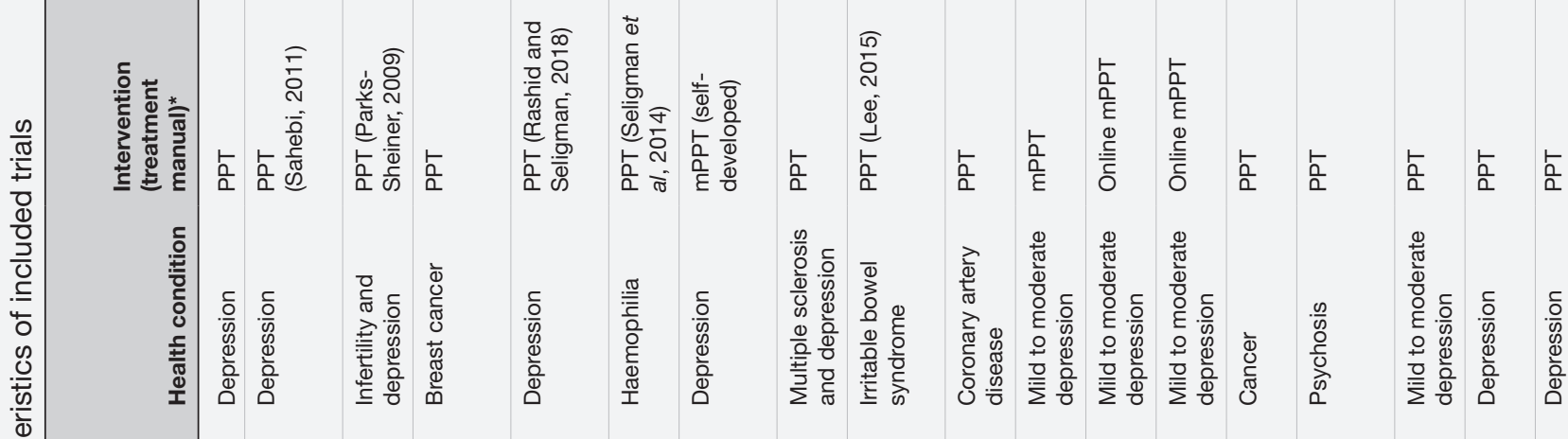

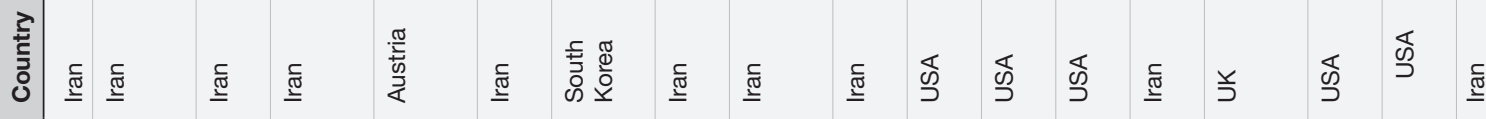

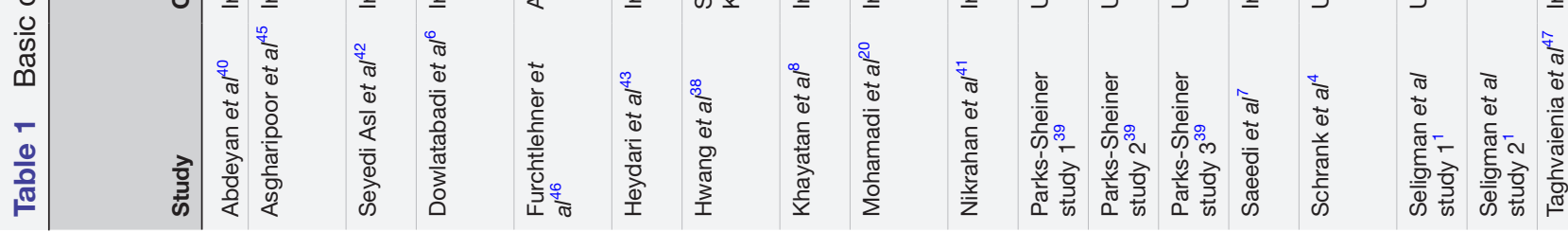



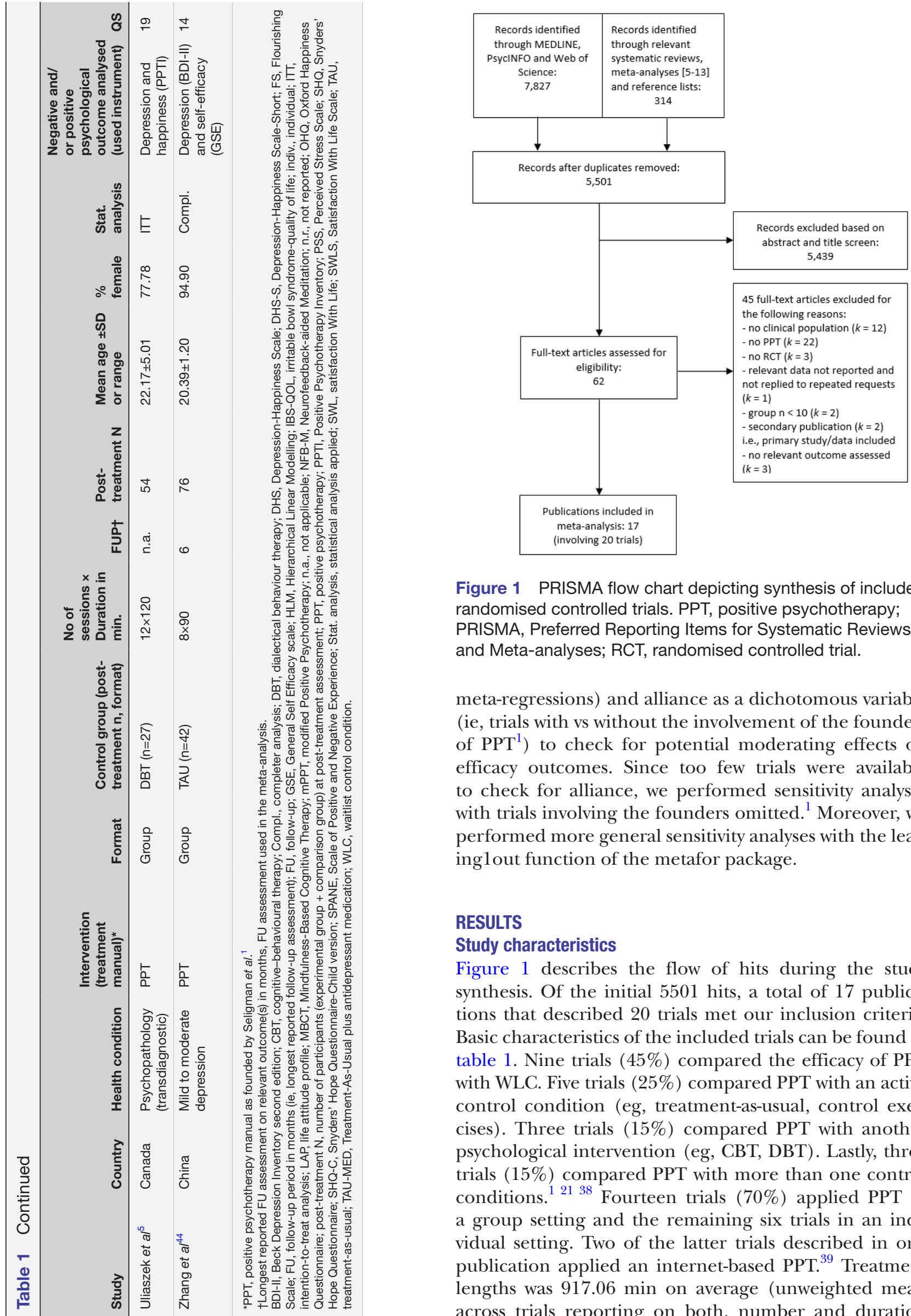

Figure 1 PRISMA flow chart depicting synthesis of included randomised controlled trials. PPT, positive psychotherapy; PRISMA, Preferred Reporting Items for Systematic Reviews and Meta-analyses; RCT, randomised controlled trial.

meta-regressions) and alliance as a dichotomous variable (ie, trials with vs without the involvement of the founders of $\mathrm{PPT}^{1}$ ) to check for potential moderating effects on efficacy outcomes. Since too few trials were available to check for alliance, we performed sensitivity analyses with trials involving the founders omitted. ${ }^{1}$ Moreover, we performed more general sensitivity analyses with the leavinglout function of the metafor package.

\section{RESULTS}

\section{Study characteristics}

Figure 1 describes the flow of hits during the study synthesis. Of the initial 5501 hits, a total of 17 publications that described 20 trials met our inclusion criteria. Basic characteristics of the included trials can be found in table 1. Nine trials $(45 \%)$ compared the efficacy of PPT with WLC. Five trials $(25 \%)$ compared PPT with an active control condition (eg, treatment-as-usual, control exercises). Three trials $(15 \%)$ compared PPT with another psychological intervention (eg, CBT, DBT). Lastly, three trials $(15 \%)$ compared PPT with more than one control conditions. ${ }^{12138}$ Fourteen trials $(70 \%)$ applied PPT in a group setting and the remaining six trials in an individual setting. Two of the latter trials described in one publication applied an internet-based PPT. ${ }^{39}$ Treatment lengths was $917.06 \mathrm{~min}$ on average (unweighted mean across trials reporting on both, number and duration 
of sessions, $\mathrm{k}=17$ ) with an SD of $374.79 \mathrm{~min}$. Note that the pioneering manual of Seligman et al constitutes of a $720 \mathrm{~min}$ (ie, 12 sessions á $60 \mathrm{~min}$ ). Average number of sessions was $9.17(\mathrm{SD}=2.71)$ and average session length was $101.76 \mathrm{~min}(\mathrm{SD}=22.03)$. Ten trials $(50 \%)$ conducted follow-up assessments on relevant outcomes whereas nine trials failed to do so. The remaining study assessed data on a relevant outcome 2 weeks after the post-treatment assessment, ${ }^{40}$ which we excluded from the follow-up data due to too short amount of time between postassessment and follow-up assessment. The average follow-up period was 7.10 months $(\mathrm{SD}=4.21)$. Most trials were conducted in Iran $(\mathrm{k}=10)$ and the USA $(\mathrm{k}=5)$. The remaining trials were conducted in Austria $(\mathrm{k}=1)$, South Korea $(\mathrm{k}=1)$, Canada $(\mathrm{k}=1)$, China $(\mathrm{k}=1)$ and the UK $(\mathrm{k}=1)$. One publication entailing three trials was a $\mathrm{PhD}$ dissertation, ${ }^{39}$ whereas the remaining trials constituted articles published in peerreviewed journals. Study quality was moderate overall with a mean of 17.85 out of the possible range from 0 to 27. Study quality varied considerably across included trials with an SD of 4.69. The detailed quality assessment per trial can be found in table 2 .

\section{Participant characteristics}

Basic characteristics of included participants per trial can be found in table 1. A total of 1360 participants participated in the included trials. Most of the participants were female (unweighted mean across included trials $=71.75 \%$ ) with a range from $23.63 \%{ }^{41}$ to $100 \% .{ }^{42}$ The patients had a pooled weighted mean age of 39.97 with a pooled SD of 10.18. It is worth noting, however, that several studies only reported age ranges rather than means and $\mathrm{SD}^{43}$ or did not report on age altogether. ${ }^{39}$

\section{The efficacy of PPT in increasing positive outcomes}

Results on the efficacy of PPT are displayed in table 3. In terms of increasing various positive outcomes such as satisfaction with life (SWL) and happiness, PPT was found moderately more effective than WLC at posttreatment assessment ( $\mathrm{g}=-0.72,95 \%$ CI: -1.31 to -0.14 , $\mathrm{k}=10, \mathrm{NNT}=2.55$ ). See figure 2 for the corresponding forest plot. Results remained similar, when the results of Mohamadi et $a l^{20}$ were entered as reported in their publication ( $\mathrm{g}=-0.82,95 \%$ CI: -1.39 to $-0.25, \mathrm{k}=10$, $\mathrm{NNT}=2.27$ ). Number of available trials allowed for a publication bias check. While a visual inspection of the funnel plot led to the suspicion of publication bias (ie, missing trials to the left and a potential outlier to the far left, see online supplemental eFigure 1), Egger's test did not indicate significant asymmetry $(t=-1.91, \mathrm{p}=0.093)$. The sensitivity analysis yielded that one trial had particular influence on the pooled effect size. When Abdeyan et al (ie, assessed positive outcome=hope) was omitted, pooled effect size decreased to $\mathrm{g}=-0.44$ (see online supplemental eTable 1). No evidence was found for the efficacy of PPT in increasing positive outcomes compared with WLC at follow-up assessment $(\mathrm{g}=-0.36,95 \% \mathrm{CI}$ : -0.83 to $0.11, k=4, N N T=5.01)$. See online supplemental
eFigure 2 for the corresponding forest plot. Follow-up assessment results are to be scrutinised with due caution in the light of low number of available trials $(\mathrm{k}=4)$, large heterogeneity in effect sizes $\left(\mathrm{I}^{2}=74.34\right)$ and the wide range of the prediction interval $(\mathrm{PI}=-1.29 ; 0.57)$. Satisfaction with life was the only positive outcome with enough trials to warrant a meta-analytical subanalysis. In comparison to WLC at post-treatment assessment, PPT was not found more effective in increasing satisfaction with life $(\mathrm{g}=-0.15,95 \%$ CI: -0.40 to $0.09, \mathrm{k}=4, \mathrm{NNT}=11.55)$. See online supplemental eFigure 3 for the corresponding forest plot. Heterogeneity in outcomes was low $\left(\mathrm{I}^{2}=11.20\right)$. The sensitivity analysis did not yield that one of the four studies was particularly influential on the pooled effect with all leaving1out analyses yielding a non-significant pooled g (see online supplemental eTable 1). In comparison to active control conditions (ie, treatment-as-usual and placebo exercises) at post-treatment assessment, PPT yielded a large effect size in increasing positive outcomes $(\mathrm{g}=-0.92,95 \%$ CI: -1.74 to $-0.11, \mathrm{k}=6, \mathrm{NNT}=2.05)$. See online supplemental eFigure 4 for the corresponding forest plot. However, heterogeneity in outcomes was large $\left(\mathrm{I}^{2}=92.51\right)$ and the prediction interval included the null ( $\mathrm{PI}=-2.98$ to 1.13 ) illustrating large variability in findings. When compared with other active treatment conditions (ie, CBT, DBT, MBCT and Neurofeedback-aided Meditation), no differences in efficacy at post-treatment assessment were found for increasing positive outcomes ( $\mathrm{g}=-0.29,95 \%$ CI: -0.89 to $0.32, \mathrm{k}=6, \mathrm{NNT}=6.24)$. See online supplemental eFigure 5 for the corresponding forest plot. Again, heterogeneity in outcomes was large $\left(\mathrm{I}^{2}=79.57\right)$ and the prediction interval included the null (PI=-1.71 to 1.13). Results remained insignificant when results of Mohamadi et $a l^{20}$ were entered as reported in their publication ( $\mathrm{g}=-0.65,95 \% \mathrm{CI}:-1.31$ to $0.01, \mathrm{k}=6$ ). Lastly, when trials with alliance (ie, involvement of the founder) were omitted, results for the comparison with WLC at post-treatment assessment remained similar ( $\mathrm{g}=-1.04,95 \%$ CI: -1.79 to $-0.28, \mathrm{k}=7, \mathrm{NNT}=1.87$, see table 3$)$.

\section{The efficacy of PPT in decreasing negative outcomes}

PPT was found moderately more effective in reducing depression, negative affect and stress than WLC at posttreatment assessment $(\mathrm{g}=0.48,95 \%$ CI: 0.18 to 0.78 , $\mathrm{k}=8$ ). See figure 2 for the corresponding forest plot. To avoid one adverse event (ie, depression, negative affect or stress), a little less than four patients needed to be treated $(\mathrm{NNT}=3.76)$. The sensitivity analysis did not yield that one of the eight studies was particularly influential on the pooled effect with all leaving1 lout analyses yielding moderate pooled effect sizes between 0.40 and 0.58 (see online supplemental eTable 1). Results on decreasing depression were similar ( $\mathrm{g}=0.57,95 \%$ CI: 0.21 to 0.92 , $\mathrm{k}=6, \mathrm{NNT}=3.22$ ). See online supplemental eFigure 6 for the corresponding forest plot. Again, the sensitivity analysis did not yield that one of the six studies was particularly influential with moderate pooled effect sizes between 0.47 


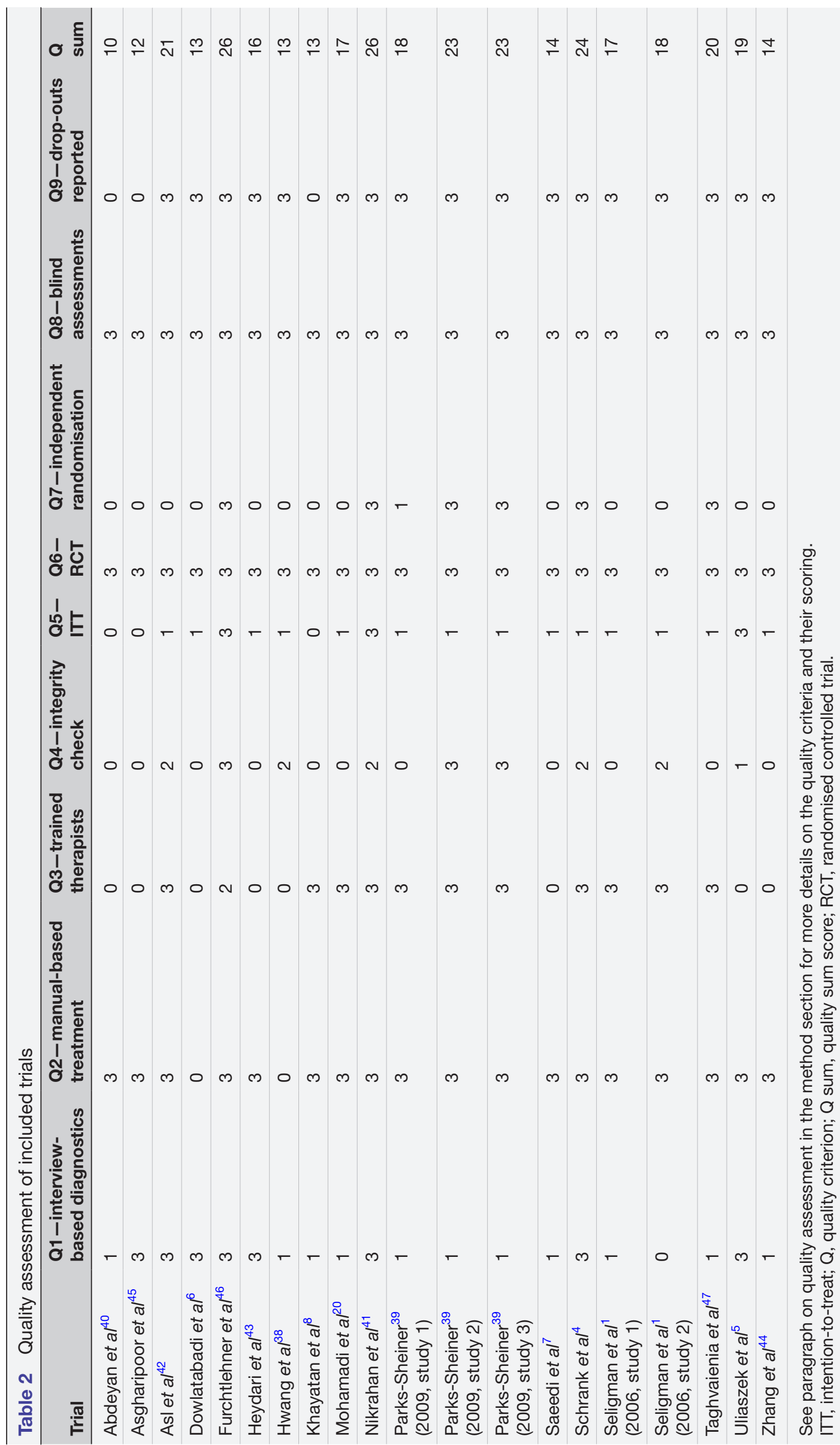


Table 3 Efficacy of PPT for increasing positive outcomes and decreasing negative outcomes

\begin{tabular}{llllllll}
$\begin{array}{l}\text { Comparison groups and timepoint of } \\
\text { assessment (ie, post vs FU) }\end{array}$ & k & g† & SE & $\begin{array}{l}95 \% \mathrm{Cl} \\
\mathrm{PI}\end{array}$ & $\mathrm{I}^{2}$ & NNT \\
\hline All trials & & & & &
\end{tabular}

Positive outcomes merged

(ie, SWL, happiness, well-being, hope, positive affect, quality of life, self-efficacy and meaning in life)

\begin{tabular}{|c|c|c|c|c|c|c|}
\hline PPT vs WLC at post & 10 & $-0.72^{\star}$ & 0.30 & $\begin{array}{l}-1.31 \text { to }-0.14 \\
\mathrm{PI}-2.55 \text { to } 1.10\end{array}$ & $90.37^{\star \star \star}$ & 2.55 \\
\hline PPT vs WLC at FU & 4 & -0.36 & 0.24 & $\begin{array}{l}-0.83 \text { to } 0.11 \\
\mathrm{PI}-1.29 \text { to } 0.57\end{array}$ & $74.34^{*}$ & 5.01 \\
\hline PPT vs ACC at post & 6 & $-0.92^{\star}$ & 0.41 & $\begin{array}{l}-1.74 \text { to }-0.11 \\
\mathrm{PI}-2.98 \text { to } 1.13\end{array}$ & $92.51^{\star \star \star}$ & 2.05 \\
\hline PPT vs ACC at FU & \multicolumn{6}{|c|}{ n.a. $(k=2)$} \\
\hline PPT vs OtherATC at post & 6 & -0.29 & 0.31 & $\begin{array}{l}-0.89 \text { to } 0.32 \\
\mathrm{PI}-1.71 \text { to } 1.13\end{array}$ & $79.57^{\star \star \star}$ & 6.24 \\
\hline PPT vs OtherATC at FU & \multicolumn{6}{|c|}{ n.a. $(k=1)$} \\
\hline \multicolumn{7}{|l|}{ ubanalyses on SWL } \\
\hline PPT vs WLC-SWL at post & 4 & -0.15 & 0.13 & $\begin{array}{l}-0.40 \text { to } 0.09 \\
\mathrm{PI}-0.45 \text { to } 0.15\end{array}$ & 11.20 & 11.55 \\
\hline
\end{tabular}

Negative outcomes merged (ie, depression, negative affect and stress)

$\begin{array}{llllll}\text { PPT vs WLC at post } & 8 & \mathbf{0 . 4 8}^{* *} & 0.15 & \begin{array}{l}0.18 \text { to } 0.78 \\ \text { PI }-0.17 \text { to } 1.13\end{array} & \begin{array}{l}51.34^{*} \\ \end{array}\end{array}$

\begin{tabular}{|c|c|c|c|c|c|c|}
\hline PPT vs WLC at FU & \multicolumn{6}{|c|}{ n.a. $(k=3)$} \\
\hline PPT vs ACC at post & \multicolumn{6}{|c|}{ All six trials conducted on depression, see below } \\
\hline PPT vs OtherATC at post & 6 & 0.08 & 0.29 & $\begin{array}{l}-0.48 \text { to } 0.64 \\
\mathrm{PI}-1.23 \text { to } 1.39\end{array}$ & $76.79^{\star \star \star}$ & 22.22 \\
\hline PPT vs OtherATC at FU & \multicolumn{6}{|c|}{ n.a. $(k=1)$} \\
\hline \multicolumn{7}{|l|}{ ubanalyses on depression } \\
\hline PPT vs WLC-depression at post & 6 & $0.57^{\star *}$ & 0.18 & $\begin{array}{l}0.21 \text { to } 0.92 \\
\mathrm{PI}-0.18 \text { to } 1.31\end{array}$ & 61.33 & 3.22 \\
\hline PPT vs WLC-depression at FU & \multicolumn{6}{|c|}{ n.a. $(k=3)$} \\
\hline PPT vs ACC-depression at post & 6 & $0.94^{\star}$ & 0.39 & $\begin{array}{l}0.18 \text { to } 1.70 \\
\mathrm{PI}-0.96 \text { to } 2.83\end{array}$ & $90.28^{\star * \star}$ & 2.03 \\
\hline
\end{tabular}
PPT vs ACC-depression at FU
n.a. $(k=3)$
PPT vs OtherATC-depression at post
n.a. $(k=3)$

Main-analyses with Seligman et al $^{1}$ and Parks-Sheiner ${ }^{39}$ omitted (ie, alliance)

Positive outcomes merged

\begin{tabular}{|c|c|c|c|c|c|c|}
\hline PPT vs WLC at post & 7 & $-1.04^{\star \star}$ & 0.38 & $\begin{array}{l}-1.79 \text { to }-0.28 \\
\mathrm{PI}-3.04 \text { to } 0.97\end{array}$ & 88.21 & 1.87 \\
\hline PPT vs ACC at post & \multicolumn{6}{|c|}{ n.a. $(k=3)$} \\
\hline PPT vs OtherATC at post & \multicolumn{6}{|c|}{ n.a. (ie, no trials with alliance) } \\
\hline \multicolumn{7}{|l|}{ legative outcomes merged } \\
\hline PPT vs WLC at post & 5 & $0.63^{\star *}$ & 0.22 & $\begin{array}{l}0.20 \text { to } 1.07 \\
\mathrm{PI}-0.14 \text { to } 1.41\end{array}$ & 44.80 & 2.89 \\
\hline
\end{tabular}
PPTvs ACC at post
n.a. $(k=3)$
PPT vs OtherATC at post
n.a. (ie, no trials with alliance)

Bold font indicates statistical significance of respective effect size.

${ }^{*} \mathrm{P}<0.05,{ }^{* *} \mathrm{p}<0.01,{ }^{* * *} \mathrm{p}<0.001$.

†A negative Hedges' $g$ for positive outcomes indicates efficacy in favour of PPT over control conditions (and vice versa). A positive Hedges' $g$ for negative outcomes indicates efficacy in favour of PPT over control conditions (and vice versa).

ACC, Activecontrol conditions; FU, follow-up; $I^{2}$, measure of heterogeneity in \% including the $\mathrm{p}$-value of the Q-statistic as indicated by asterisks; $\mathrm{k}$, number of trials for the respective comparison; n.a., not applicable; NNT, numbers needed to treat; OtherATC, Other Active Treatment Conditions (included Cognitive Behavioral Therapy, Dialectic Behavioral Therapy and Mindfulness-Based Cognitive Behavioral Therapy); PI, prediction interval; post, post-treatment assessment; PPT, positive psychotherapy; SWL, Satisfaction With Life; WLC, Waitlist Control conditions. 

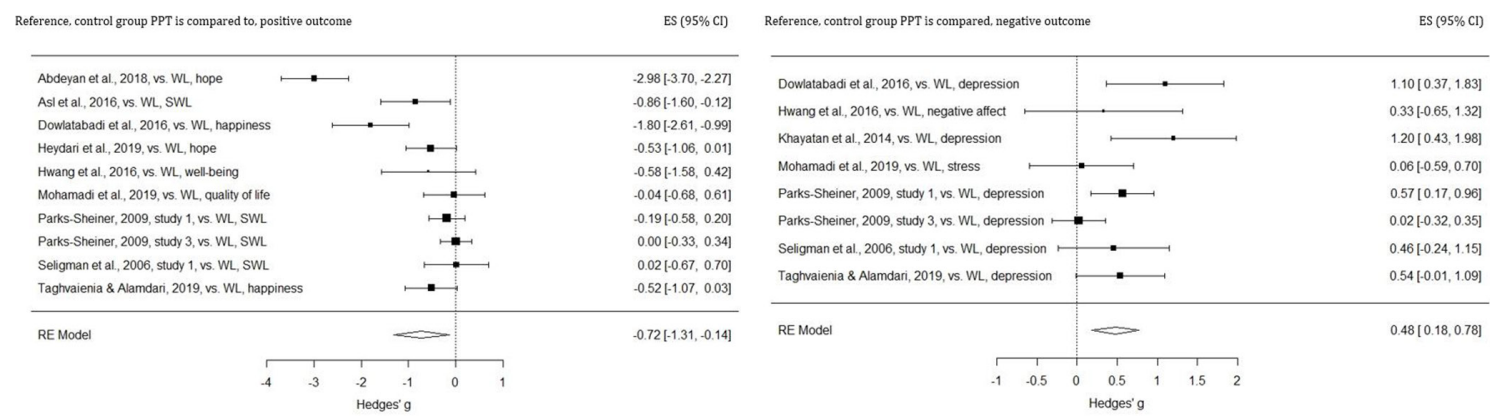

Figure 2 Forest plots - efficacy of PPT versus waitlist control (WC) conditions in increasing positive (left) and decreasing negative (right) psychological outcomes at post-treatment assessment. PPT, positive psychotherapy.

and 0.68 for the leaving1out analyses (see online supplemental eTable 1). Prediction intervals for both analyses (ie, all negative outcomes and depression only) excluded the null ( $\mathrm{PI}=-0.17$ to 1.13 ; $\mathrm{PI}=-0.18$ to 1.31 , respectively) highlighting substantial levels of heterogeneity in efficacy outcomes and remaining uncertainty about the true efficacy when similar future trials accumulate. In comparison to active control conditions (ie, treatment-as-usual with or without medication and placebo exercises) at posttreatment assessment, PPT yielded large effect sizes in reducing depression ( $\mathrm{g}=0.94,95 \% \mathrm{CI}$ : 0.18 to $1.70, \mathrm{k}=6$, $\mathrm{NNT}=2.03)$. Please find the corresponding forest plot in online supplemental eFigure 7. Again, heterogeneity was large $\left(\mathrm{I}^{2}=90.28\right)$ and the PI excluded the null $(P I=-0.96$ to $2.83)$. When compared with other active treatment conditions (ie, CBT, DBT, MBCT and Neurofeedback-aided Meditation), no differences in efficacy at post-treatment assessment were found for decreasing negative outcomes ( $\mathrm{g}=0.08,95 \%$ CI: -0.48 to $0.64, \mathrm{k}=6, \mathrm{NNT}=22.22$ ). Please find the corresponding forest plot in online supplemental eFigure 8. Trials that included follow-up assessments on the efficacy of PPT in decreasing negative outcomes were too few to allow for meta-analytical review for all included comparisons $(k<4)$. Lastly, when trials with alliance (ie, involvement of the founder) were omitted, results for the comparison with WLC at post-treatment assessment remained similar ( $\mathrm{g}=0.63,95 \%$ CI: 0.20 to $1.07, \mathrm{k}=5$, $\mathrm{NNT}=2.89$, see table 3 ).

\section{Moderator analyses}

Moderator analyses revealed that trial quality as a continuous variable was associated with effect sizes in most of the above-mentioned analyses. See table 4 for an overview of results. With regard to the efficacy of PPT in increasing positive outcomes in comparison to WLC at post-treatment assessment, trial quality was found to be a significant moderator with higher trial quality being associated with larger effect sizes $(b=0.17, p=0.003)$. A similar result was found for the follow-up assessment results $(b=0.12, p=0.036)$. In terms of the comparison with active control conditions at post-treatment assessment, trial quality was also found to moderate effect sizes with higher trial quality being associated with larger effect sizes $(b=0.18, p=0.015)$. No significant moderation of trial quality was found for the comparison with other active treatment conditions $(b=-0.01, p=0.907)$ nor for the subanalysis on satisfaction with life $(\mathrm{b}=-0.01, \mathrm{p}=0.915)$.

In terms of the efficacy of PPT in decreasing negative outcomes in comparison to WLC at post-treatment assessment, trial quality was found to be a significant moderator with higher trial quality being associated with smaller effect sizes $(b=-0.08, p=0.003)$. A similar result was found for the sub-analyses on depression $(b=-0.11$, $\mathrm{p}<0.001)$. Similarly, the subanalysis on depression for the comparison of PPT and active control conditions yielded a significant moderation of trial quality with higher trial quality being associated with smaller effect sizes $(b=-0.17$, $\mathrm{p}=0.005$ ). However, a significant moderation was found for the comparison with other active treatment conditions with higher trial quality being related to larger effect sizes in decreasing negative outcomes $(b=0.13, p<0.001)$. No evidence was found for a moderation of treatment length in any of the analyses (see table 4).

\section{DISCUSSION}

Our systematic search resulted in 20 RCTs that assessed the efficacy of PPT. The results of the meta-analysis indicate that PPT can effectively increase positive psychological outcomes and decrease depression at post-treatment assessment. Both comparisons with WLC and active control groups support the short-term efficacy of PPT. Overall, there is too few data on the long-term efficacy of PPT. Additionally, moderator analyses yielded that trial quality was significantly associated with effect size. For positive outcomes, higher quality of trials was related to larger effect sizes. Whereas for depression, higher quality of trials was related to smaller effect sizes. However, the low number of available trials, large heterogeneities, identification of some influential single trials in the sensitivity analyses and wide prediction intervals call for cautious statements on the efficacy.

The findings support the short-term efficacy of PPT in increasing positive psychological outcomes. However, the larger magnitude in effect sizes for comparisons with active control conditions (pooled $\mathrm{g}=-0.92$ ) compared with WLC (pooled $g=-0.72$ ) is surprising and 
Table 4 Subanalyses on trial quality and treatment length as potential moderators

\begin{tabular}{|c|c|c|c|c|c|}
\hline Comparison groups and time point of assessment & k & Intercept & b & Rem. $I^{2}$ & $P$ value \\
\hline \multicolumn{6}{|c|}{ Potential moderator: trial quality } \\
\hline \multicolumn{6}{|c|}{ Positive outcomes merged (eg, happiness, SWL, hope, quality of life) } \\
\hline PPT vs WLC at post & 10 & -3.60 & 0.17 & $79.93^{\star \star \star}$ & 0.003 \\
\hline PPT vs WLC at follow-up & 4 & -2.56 & 0.12 & 38.01 & 0.036 \\
\hline PPT vs ACC at post & 6 & -4.21 & 0.18 & $83.61^{\star \star *}$ & 0.015 \\
\hline PPT vs OtherATC at post & 6 & -0.13 & -0.01 & $82.40^{\star * \star}$ & 0.907 \\
\hline \multicolumn{6}{|l|}{ Sub-analysis on SWL } \\
\hline PPT vs WLC at post & 4 & -0.02 & -0.01 & 56.42 & 0.915 \\
\hline \multicolumn{6}{|c|}{ Negative outcomes merged (ie, depression, negative affect and stress) } \\
\hline PPT vs WLC at post & 8 & 2.00 & -0.08 & 0 & 0.003 \\
\hline PPT vs ACC at post & \multicolumn{5}{|c|}{ All six trials conducted on depression, see below } \\
\hline PPT vs OtherATC at post & 6 & -2.24 & 0.13 & 21.28 & $<0.001$ \\
\hline \multicolumn{6}{|l|}{ Subanalysis on depression } \\
\hline PPT vs WLC at post & 6 & 2.50 & -0.11 & 0 & $<0.001$ \\
\hline PPT vs ACC at post & 6 & 4.47 & -0.17 & $76.91^{\star * \star}$ & 0.005 \\
\hline \multicolumn{6}{|l|}{ Potential Moderator: Treatment length $\dagger$} \\
\hline \multicolumn{6}{|c|}{ Positive outcomes merged (eg, happiness, SWL, hope, quality of life) } \\
\hline PPT vs WLC at post & 9 & -1.19 & 0.00 & 89.69 & 0.734 \\
\hline PPT vs WLC at follow-up & n.a. $(k=3)$ & & & & \\
\hline PPT vs ACC at post & n.a. $(k=3)$ & & & & \\
\hline PPT vs OtherATC at post & 6 & 1.16 & -0.00 & 74.95 & 0.159 \\
\hline
\end{tabular}

Subanalysis on SWL

PPT vs WLC at post

n.a. $(k=3)$

Negative outcomes merged (ie, depression, negative affect and stress)

$\begin{array}{lccccc}\text { PPT vs WLC at post } & 7 & 0.92 & -0.00 & 16.70 & 0.368 \\ \text { PPT vs ACC at post } & \text { n.a. }(k=3) & & & & \\ \text { PPT vs OtherATC at post } & 6 & -0.98 & 0.00 & 74.26 & 0.285 \\ \text { Subanalysis on depression } & & & & & \\ \text { PPT vs WLC at post } & 5 & 0.82 & -0.00 & 21.67 & 0.801\end{array}$

Bold font indicates statistical significance of moderation.

${ }^{*} \mathrm{P}<0.05,{ }^{* *} \mathrm{p}<0.01,{ }^{* \star *} \mathrm{p}<0.001$.

†Number of trials differs in comparison to main-analyses since not all publications reported on treatment length as can be witnessed in table 1.

ACC, active control condition; $b$, refers to the interaction term between treatment and covariate (in Hedges' g); rem. $I^{2}$, remaining amount of unexplained heterogeneity including the $\mathrm{p}$-value of the Q-statistic as indicated by asterisks; OtherATC, Other Active Treatment Condition; post, post-treatment assessment; PPT, positive psychotherapy; SWL, satisfaction with life; ; WLC, waitlist control conditions.

counterintuitive. Usually the opposite pattern is found in clinical research. ${ }^{21}{ }^{28}$ Unplanned post hoc investigations on potential reasons hint towards the effect of an almost outlier in the analysis involving active comparison groups. ${ }^{7}$ This trial offered either PPT or treatmentas-usual to patients with cancer and yielded a strikingly large effect size at post-treatment assessment favouring PPT $(g=-2.79)$ for increasing meaning in life. Furthermore, a second trial on cancer patients also produced a large effect size for increasing happiness $(\mathrm{g}=-1.80)$ as compared with waitlist at post-treatment assessment. ${ }^{6}$ While these two trials on cancer patients suggest that PPT might be highly effective in increasing positive outcomes in this population, two trials remain of course a slim evidence base. It should be noted, however, that the analysis on passive control conditions (ie, waitlist controls) also involved an almost outlier. ${ }^{40}$ This study offered PPT to depressed patients and yielded a strikingly large effect size at post-treatment assessment $(\mathrm{g}=-2.98)$ favouring PPT in increasing hope. Both almost outlier studies involved a moderate sample size (see table 1). All this suggests that more trials are needed to allow for firmer conclusions.

When PPT was compared with other established psychological interventions such as CBT, current data did 
not suggest any significant difference in efficacy. Accordingly, the results of the six RCTs included in this comparison suggests that PPT is similarly effective in increasing positive psychological outcomes. However, due to the low number of trials for this comparison these findings need to be viewed with due caution.

The first and foremostly assessed negative outcome in the PPT literature remains depression. As suggested and intended by its developers, PPT was found moderately to largely effective in lowering depressive symptoms. Again, the counterintuitive pattern was found with larger effect sizes in lowering depression for PPT in comparison to active control conditions (pooled $\mathrm{g}=0.94$ ) as opposed to WLC (pooled $\mathrm{g}=0.57$ ). Once more, unplanned post hoc investigations were performed in an attempt to find potential reasons for the counterintuitive finding. Again, we found that an almost outlier might explain the difference. The analysis involving active control groups involved an almost outlier with an effect size of $\mathrm{g}=2.45,{ }^{44}$ whereas the analysis involving WLC did not involve such an almost outlier.

Data on the efficacy at follow-up assessments altogether were scarce. The only feasible analysis on follow-up assessment data (ie, PPT vs WLC in increasing positive outcomes) yielded a non-significant effect size. The current available literature does not allow for any other valid follow-up analyses and, thus, conclusions on the long-term efficacy of PPT cannot not yet be made. This represents perhaps the main limitation of the literature on the efficacy of PPT. For the same reason, additional sensitivity analyses (eg, group vs individual PPT, or PPT efficacy by health condition vs mental health condition) were not feasible.

Trial quality overall was moderate and, therefore, leaves room for improvement. Results overall are comparable to related meta-analyses on positive psychology interventions (PPIs) more generally which report moderate effect sizes in increasing positive outcomes and decreasing negative outcomes. ${ }^{11-19}$ A recent meta-analysis on PPIs further also reports on a significant relation between trial quality and the efficacy of PPIs. ${ }^{15}$ However, PPIs vary considerably and generalisations from meta-analyses on PPIs on PPT are, therefore, not straightforward.

This represents the first meta-analysis with an exclusive focus on the efficacy of PPT. Several limitations need to be considered. First and foremost, the number of included trials is relatively small and accordingly more research is needed to draw firmer conclusions. Second, depression and SWL were the only two outcomes with enough trials to warrant subanalyses. More research is needed to allow for more homogeneous analyses on PPT efficacy for specific outcomes. Third and related to the second limitation, the two overarching analyses on various positive and negative outcomes involved large heterogeneity, respectively. The decision to conduct such overarching analyses on heterogeneous outcomes was based on the overall scarcity of trials. We aimed at conducting more homogeneous subanalyses were possible which were, as mentioned, only feasible for depression and SWL. As more trials accumulate, more fine-grained analyses will become feasible. Fourthly and lastly, the long-term efficacy of PPT remains uncertain due to lack of follow-up assessments.

\section{Conclusion}

Our findings indicate that PPT can effectively increase positive outcomes and decrease negative outcomes at posttreatment assessment. However, there is lack of follow-up data and the number of available trials altogether remains scarce precluding many of the planned subanalyses. More research with high methodological rigour and including follow-up assessments is needed to draw firmer and more precise conclusions on PPT efficacy.

\section{Twitter Thole Hilko Hoppen @HoppenDr}

Contributors THH and NM conceptualised the meta-analysis conducted the systematic literature search and coding of studies. THH performed the statistical analyses. THH and NM wrote the manuscript and agreed to be accountable for all aspects of the work.

Funding The authors have not declared a specific grant for this research from any funding agency in the public, commercial or not-for-profit sectors.

Competing interests None declared.

Patient consent for publication Not required.

Provenance and peer review Not commissioned; externally peer reviewed.

Data availability statement Data sharing not applicable as no datasets generated and/or analysed for this study. We performed a meta-analysis on published und publicly accessible data. No additional data available.

Supplemental material This content has been supplied by the author(s). It has not been vetted by BMJ Publishing Group Limited (BMJ) and may not have been peer-reviewed. Any opinions or recommendations discussed are solely those of the author(s) and are not endorsed by BMJ. BMJ disclaims all liability and responsibility arising from any reliance placed on the content. Where the content includes any translated material, BMJ does not warrant the accuracy and reliability of the translations (including but not limited to local regulations, clinical guidelines, terminology, drug names and drug dosages), and is not responsible for any error and/or omissions arising from translation and adaptation or otherwise.

Open access This is an open access article distributed in accordance with the Creative Commons Attribution Non Commercial (CC BY-NC 4.0) license, which permits others to distribute, remix, adapt, build upon this work non-commercially, and license their derivative works on different terms, provided the original work is properly cited, appropriate credit is given, any changes made indicated, and the use is non-commercial. See: http://creativecommons.org/licenses/by-nc/4.0/.

\section{ORCID iDs}

Thole Hilko Hoppen http://orcid.org/0000-0002-6050-8696

Nexhmedin Morina http://orcid.org/0000-0002-2331-9140

\section{REFERENCES}

1 Seligman MEP, Rashid T, Parks AC. Positive psychotherapy. Am Psychol 2006;61:774-88.

2 Peterson C, Seligman MEP. Character strengths and virtues: a Handbook and classification. Oxford University Press, 2004

3 Walsh S, Cassidy M, Priebe S. The application of positive psychotherapy in mental health care: a systematic review. J Clin Psychol 2017;73:638-51.

4 Schrank B, Brownell T, Jakaite Z, et al. Evaluation of a positive psychotherapy group intervention for people with psychosis: pilot randomised controlled trial. Epidemiol Psychiatr Sci 2016;25:235-46.

5 Uliaszek AA, Rashid T, Williams GE, et al. Group therapy for university students: a randomized control trial of dialectical behavior therapy and positive psychotherapy. Behav Res Ther 2016;77:78-85.

6 Dowlatabadi MM, Ahmadi SM, Sorbi MH, et al. The effectiveness of group positive psychotherapy on depression and happiness in breast 
cancer patients: a randomized controlled trial. Electron Physician 2016;8:2175-80.

7 Saeedi B, Khoshnood Z, Dehghan M, et al. The effect of positive psychotherapy on the meaning of life in patients with cancer: a randomized clinical trial. Indian J Palliat Care 2019;25:210.

8 Khayatan T, Azkhosh M, Bahmani B. Group positive psychotherapy and depression of females affected by multiple sclerosis. Iran Rehabil J 2014;12:49-53.

9 Rashid T, Seligman MP. Positive psychotherapy: clinician manual. Oxford University Press, 2018.

10 Moher D, Liberati A, Tetzlaff J, et al. Preferred reporting items for systematic reviews and meta-analyses: the PRISMA statement. Ann Intern Med 2009;151:264-9.

11 Bolier L, Haverman M, Westerhof GJ, et al. Positive psychology interventions: a meta-analysis of randomized controlled studies. BMC Public Health 2013;13:119.

12 Chakhssi F, Kraiss JT, Sommers-Spijkerman M, et al. The effect of positive psychology interventions on well-being and distress in clinical samples with psychiatric or somatic disorders: a systematic review and meta-analysis. BMC Psychiatry 2018;18:211.

13 Davis DE, Choe E, Meyers J, et al. Thankful for the little things: a meta-analysis of gratitude interventions. J Couns Psychol 2016;63:20-31.

14 Hendriks T, Schotanus-Dijkstra M, Hassankhan A. The efficacy of positive psychological interventions from non-Western countries: a systematic review and meta-analysis. IJW 2018;8:71-98.

15 Hendriks T, Schotanus-Dijkstra M, Hassankhan A, et al. The efficacy of multi-component positive psychology interventions: a systematic review and meta-analysis of randomized controlled trials. $J$ Happiness Stud 2020;21:357-90.

16 Hendriks T, Warren MA, Schotanus-Dijkstra M, et al. How WEIRD are positive psychology interventions? A bibliometric analysis of randomized controlled trials on the science of well-being. J Posit Psychol 2019;14:489-501.

17 Sin NL, Lyubomirsky S. Enhancing well-being and alleviating depressive symptoms with positive psychology interventions: a practice-friendly meta-analysis. J Clin Psychol 2009;65:467-87.

18 Casellas-Grau A, Font A, Vives J. Positive psychology interventions in breast cancer. A systematic review. Psychooncology 2014;23:9-19.

19 White CA, Uttl B, Holder MD. Meta-Analyses of positive psychology interventions: the effects are much smaller than previously reported. PLoS One 2019;14:e0216588.

20 Mohamadi J, Ghazanfari F, Drikvand FM. Comparison of the effect of dialectical behavior therapy, mindfulness based cognitive therapy and positive psychotherapy on perceived stress and quality of life in patients with irritable bowel syndrome: a pilot randomized controlled trial. Psychiatr Q 2019;90:565-78.

21 Cuijpers P, van Straten A, Bohlmeijer E, et al. The effects of psychotherapy for adult depression are overestimated: a meta-analysis of study quality and effect size. Psychol Med 2010;40:211-23.

22 Smit Y, Huibers MJH, loannidis JPA, et al. The effectiveness of longterm psychoanalytic psychotherapy--a meta-analysis of randomized controlled trials. Clin Psychol Rev 2012;32:81-92.

23 Morina N, Koerssen R, Pollet TV. Interventions for children and adolescents with posttraumatic stress disorder: a meta-analysis of comparative outcome studies. Clin Psychol Rev 2016;47:41-54.

24 R Core Team. R. a language and environment for statistical computing, 2019. Available: http://www.R-project.org/

25 Viechtbauer W. Conducting Meta-Analyses in $R$ with the metafor Package. J Stat Softw 2010;36:1-48.

26 Borenstein M, Hedges LV, Higgins JPT. Introduction to meta-analysis. John Wiley \& Sons, 2011.
27 Lipsey MW, Wilson DB. Practical meta-analysis, [Nachdr.]. Applied social research methods series. Thousand Oaks, Calif: SAGE Publ, 2009: 49.

28 Hoppen TH, Morina N. Is high-quality of trials associated with lower treatment efficacy? A meta-analysis on the association between study quality and effect sizes of psychological interventions for pediatric PTSD. Clin Psychol Rev 2020;78:101855.

29 Cohen J. Statistical power analysis for the behavioral sciences. 2nd ed. Hoboken: Taylor and Francis, 2013.

30 Higgins JPT, Thompson SG, Deeks JJ, et al. Measuring inconsistency in meta-analyses. BMJ 2003;327:557-60.

31 IntHout J, loannidis JPA, Rovers MM, et al. Plea for routinely presenting prediction intervals in meta-analysis. BMJ Open 2016;6:e010247.

32 Riley RD, Higgins JPT, Deeks JJ. Interpretation of random effects meta-analyses. BMJ 2011;342:d549.

33 Hunter JE, Schmidt FL. Methods of meta-analysis: correcting error and bias in research findings. London: Sage Publications, 2004.

34 Tabachnick BG, Fidell LS. Using multivariate statistics. ed. 6, 2013.

35 Sterne JAC, Sutton AJ, loannidis JPA, et al. Recommendations for examining and interpreting funnel plot asymmetry in meta-analyses of randomised controlled trials. BMJ 2011;343:d4002.

36 Duval S, Tweedie R. Trim and fill: a simple funnel-plot-based method of testing and adjusting for publication bias in meta-analysis. Biometrics 2000;56:455-63.

37 Kraemer HC, Kupfer DJ. Size of treatment effects and their importance to clinical research and practice. Biol Psychiatry 2006;59:990-6.

38 Hwang K, Kwon A, Hong C. A preliminary study of new positive psychology interventions: Neurofeedback-aided meditation therapy and modified positive psychotherapy. Curr Psychol 2017;36:683-95.

39 Parks-Sheiner AC. Positive psychotherapy: building a model of empirically supported self-help. Dissertations available from ProQuest, 2009.

40 Abdeyan T, Mahsa KK, Mohammad Z. The effect of psychosocial group based on positive psychology on Hopefulness in patients with major depressive disorder: a clinical trial. Electron J Gen Med 2018;15:em49.

41 Nikrahan GR, Laferton JAC, Asgari K, et al. Effects of positive psychology interventions on risk biomarkers in coronary patients: a randomized, wait-list controlled pilot trial. Psychosomatics 2016:57:359-68.

42 Seyedi Asl ST, Sadeghi K, Bakhtiari M, et al. Effect of group positive psychotherapy on improvement of life satisfaction and the quality of life in infertile woman. Int J Fertil Steril 2016;10:105-12.

43 Heydari MG, Shirazi M, Sanagouyemoharer GR. Evaluating the efficacy of positive psychotherapy on life expectancy in students with hemophilia: a randomized controlled trial. Pract Clin Psychol 2019;7:63-70.

44 Zhang JP, Guo Y-F, Zhang X. Effects of positive psychotherapy on depression and self-efficacy in undergraduate nursing students positive psychotherapy. Res Rev J Nurs Health Sci 2015:12-18.

45 Asgharipoor N, Asgharnejad AA, Arshadi HR. Comparative study on the effectiveness of positive psychotherapy and group cognitivebehavioral therapy for the patients suffering from major depressive disorder. Iran J Psychiatry Behav Sci 2012;2:33-41.

46 Furchtlehner LM, Schuster R, Laireiter AR. A comparative study of the efficacy of group positive psychotherapy and group cognitive behavioral therapy in the treatment of depressive disorders: a randomized controlled trial. J Posit Psychol 2019:1-14.

47 Taghvaienia A, Alamdari N. Effect of positive psychotherapy on psychological well-being, happiness, life expectancy and depression among retired teachers with depression: a randomized controlled trial. Community Ment Health J 2020;56:229-37. 